\title{
Autologous Bone Marrow Derived Stem Cells for the Treatment of Multiple Sclerosis
}

\author{
Nassim Abi Chahine ${ }^{1}$, Tarek Wehbe ${ }^{2}$, Johny Rashed ${ }^{3}$, Ramzi Hilal ${ }^{4}$, Nada Elias ${ }^{5}$ \\ ${ }^{1}$ Department of Neurosurgery, The Lebanese-Canadian Hospital, Beirut, Lebanon \\ ${ }^{2}$ Department of Hematology, The Lebanese-Canadian and Notre Dame University Hospital, Beirut, Lebanon \\ ${ }^{3}$ Department of Biology, University of Balamand, Balamand, Lebanon \\ ${ }^{4}$ Department of Neurology, The Lebanese-Canadian Hospital, Beirut, Lebanon \\ ${ }^{5}$ Neuro- rehabilitation, Physical Therapy, Beirut, Lebanon
}

Stem cell therapy, an evolving, progressive field of therapeutics has shown several successes in areas where classic treatments failed to prevent or stop disability. Starting in 2009, twenty two sequential patients with progressive Multiple Sclerosis (MS) courses were treated with Autologous Bone Marrow Mononuclear stem cells (BM-MNSCs). The cells were given both intravenously and intrathecally. Using the Expanded Disability Status Scale (EDSS) score for evaluation, our data indicates that the majority of the patients benefited on the average one point on the scale. This paper adds to the body of evidence suggesting the safety and efficacy of autologous BM-MNSCs in the treatment of MS and awaits validation through larger, randomized studies.

Keywords: Stem cells, Autologous bone marrow, Multiple Sclerosis, The expanded disability status scale, Mononuclear cells

\section{Introduction}

MS is primarily an autoimmune disease involving several arms of the cellular and humoral immune systems resulting ultimately in axonal degeneration. The disease prevalence has been on the rise in several areas around the world including the Middle East $(1,2)$.

The diagnosis requires proof of the disease activity on sequential examinations and central nervous system magnetic resonance imaging (MRI). The incidence rises particularly between the ages of 20 and 45. The etiology and

\footnotetext{
Accepted for publication October 12, 2016, Published online November 30, 2016

Correspondence to Nassim Abi Chahine

Neurosurgery, The Lebanese-Canadian Hospital, Beirut Lebanon Tel: +009613082498, Fax: +009616951100

E-mail: nassim@wp.eu

(c) This is an open-access article distributed under the terms of the Creative Commons Attribution Non-Commercial License (http://creativecommons.org/ licenses/by-nc/4.0/), which permits unrestricted non-commercial use, distribution, and reproduction in any medium, provided the original work is properly cited.
}

triggers of the disease remain poorly defined. Genetic, viral along with environmental factors are possible candidate etiologies of the disease $(3,4)$.

The effector cells include different categories of the Tlymphocytes, B-lymphocytes, Natural killer cells, and the oligodendrocytes. The deregulated immune inflammatory mediators include IFN- $\gamma$, tumor necrosis factor (TNF), and several interleukins. This unusual combination of abnormally acting cells and factors interact in a very complex schema to produce the clinical manifestations of MS (4-7).

The myelin generating, supporting and feeding cells, the oligodendrocytes, are attacked by this immune army, leading to thinning and disappearance of the myelin sheath. The degradation of myelin gradually leads to electrical instability, poor axonal conduction and functional aberrations along the whole neuron (4). This process starts with inflammation and ends with axonal degradation and dysfunction.

Depending on the location and degree of damage, the lesions show up along the white matter and its tracts in 
the CNS in order of frequency along the basal and lateral ganglia, brain stem, spinal cord, and optic nerves. The symptoms correlate with the damage at the level of the corresponding neurons. The symptoms start with weakness and tingling at random sites of the body. Muscle spasms, visual problems (nystagmus, diplopia $\cdots$ ), chronic pain, dizziness, headaches, dysarthria, dysphagia, urinary and bowel incontinence are some of those manifestations $(4,7)$.

The patterns and severity of the symptoms are grouped into four subtypes (8):

1. The Relapsing Remitting MS (RRMS): Affects almost $80 \%$ of the patients with a course of flare-ups alternating with improvements. The symptoms are dynamic and sometimes abrupt with differing degrees of recovery and periods of stability or slow progression.

2. Primary progressive MS (PPMS): The course is usually progressive despite pharmacotherapy. This type affects about $10 \%$ of the patients.

3. Secondary progressive MS (SPMS): Progressive course of symptoms after an initial course of a relapsing remitting pattern with or without periods of regression or stability.

4. Progressive relapsing MS (PRMS): The rarest type. This particular presentation shows as disease that continuously progresses and worsens.

The treatments of multiple sclerosis, which can be effective at relieving the symptoms, usually have no or little effect on the actual demyelination and immune disturbances. There is no available cure. Physicians usually start with corticosteroids (8-10). Interferons, Natalizumab, Fingolimod, Glatiramer Actetate and others may allow to hit one or more of the abnormalities found in MS rather than restore the normal immune balance and that explains the lack of full remissions or cure with all these medications carrying the price tags of several thousands of dollars yearly (9-11).

Other strategies involve attacking the leukocyte specific adhesion molecules, tipping the balance to producing immune suppressors like IL-4, IL-10 and TGF-beta, inhibiting IL-2, blocking certain specific molecules like CD20 and CD52 have been shown to control MS at least for a period of time (9-13).

The BM-MNSCs are progenitor cells with regenerative and immune modulatory and growth stimulating properties. They may induce angiogenesis and differentiate into different types of cells of the nervous system. The BM-MNSCs include the hematopoietic and mesenchymal cells found in the bone marrow in small numbers (14-16).

The BM-MNSCs have been found in several studies to be well tolerated and to have the ability to generate neu- rons, counteract fibrosis and oxidation. Those cells were considered for several reasons safe and effective. They are easy to handle technically and to inject using sterile methods (16-19).

Stem cell therapies have given the MS and neuro-regenerative fields a breath of hope with the promise of regenerating and immune modulating properties. The stem cell immune modulator properties make them capable of breaking the process that leads to MS. Stem cells have several useful properties including their ability to self-renew, remodeling, differentiation, immune modulation, and immune balance restoration. Mesenchymal stem cells are also known to be immune modulators, a property that may be of utmost importance $(20,21)$.

To quantify, monitor and evaluate the MS patients receiving this therapy we resorted to the EDSS which is a validated, reproducible tool that quantifies disability in eight Functional Systems and allows to quantitatively assign a Functional System Score in each case (22).

There has been a lot of data but a scarcity of randomized studies. In 2011, Connick et al. administered intravenous autologous bone-marrow-derived mesenchymal stem cells at a mean dose was $1.6 \times 10^{6}$ cells per $\mathrm{kg}$ to secondary progressive MS patients in an open-label study. They noted improvement after treatment in visual acuity and visual evoked response latency with an increase in the optic nerve area. They concluded that it probably worked by neuro-protection (23).

\section{Patients and Methods}

Starting in 2009, patients with MS were enrolled sequentially on this study using bone marrow derived stem cells. The study was reviewed and approved by the scientific committee and Institutional Review Board at the hospital. The patients had to have confirmed disease by 2 neurologists based on the clinical picture, the MRI showing progression on two occasions, and the presence of change over time correlating with the MRI findings. The patients had also to have progressive disease despite treatment using at least 2 standard lines of therapy and have adequate renal, hepatic, pulmonary and cardiac functions. All patients signed an informed consent and received full explanation of the protocol, expected outcomes and adverse events.

The patients were given 3 days of Granulocyte-colony stimulating factor at $10 \mathrm{mg} / \mathrm{kg}$ divided in 2 equal doses prior to bone marrow collection. On the day of the procedure, the bone marrow was collected under sterile conditions under local or general anesthesia and adequate 
sedation. About three milliliters per $\mathrm{kg}$ of marrow were collected from the posterior iliac crests bilaterally. Six access sites were used to aspirate the marrow. The marrow was aspirated using a gauge 8 Jamshidi needle into a syringe containing heparin sodium at $10 \%$ concentration. The collected BM-MNSCs were separated using density gradient centrifugation before counting the cells using a Neubauer chamber. The cells were subjected to microbiologic, microscopic, and serologic testing. The resulting cells were re-suspended to prepare $5 \sim 10 \mathrm{ml} \mathrm{BM-MNSCs}$ to be injected intrathecally.

The intrathecally injected cells were adjusted to about 0.5 million mononuclear cells per kilogram of body weight at the L4 L5 level under sterile conditions and the rest of the cells were given intravenously. Table 1 shows the patients' characteristics before treatment.

\section{Results}

22 sequential patients, 10 males and 12 females aged 22 to 56 years were accrued. Seven patients were classified as having the SPMS type, eight as PPMS, and seven as RRMS. Pretreatment EDSS scores ranged between 1 and 8.5 with an average of 5.5 (Table 1).

Table 1. Details the patient demographics and pre - treatment EDSS

\begin{tabular}{|c|c|c|c|c|}
\hline $\begin{array}{l}\text { Patient } \\
\text { number }\end{array}$ & Gender & Age & $\begin{array}{c}\text { Pre-treatment } \\
\text { EDSS }\end{array}$ & Comments \\
\hline 1 & $M$ & 49 & 6 & SPMS \\
\hline 2 & $M$ & 40 & & SPMS \\
\hline 3 & $\mathrm{~F}$ & 28 & 2.5 & PPMS \\
\hline 4 & $M$ & 30 & 3 & PPMS \\
\hline 5 & $M$ & 30 & 1.5 & PPMS \\
\hline 6 & $\mathrm{~F}$ & 49 & 6 & SPMS \\
\hline 7 & $M$ & 56 & 7 & SPMS \\
\hline 8 & $\mathrm{~F}$ & 37 & 7.5 & SPMS \\
\hline 9 & $\mathrm{~F}$ & 37 & 7.5 & SPMS \\
\hline 10 & $F$ & 50 & 8.5 & PPMS \\
\hline 11 & $\mathrm{~F}$ & 27 & 8.5 & RRMS \\
\hline 12 & $M$ & 45 & 8.5 & RRMS \\
\hline 13 & $M$ & 52 & 6.5 & PPMS \\
\hline 14 & $M$ & 37 & 7.5 & SPMS \\
\hline 15 & $\mathrm{~F}$ & 40 & 2.5 & PPMS \\
\hline 16 & $\mathrm{~F}$ & 48 & 1 & PPMS \\
\hline 17 & $\mathrm{~F}$ & 35 & 2.5 & RRMS \\
\hline 18 & $\mathrm{~F}$ & 54 & 4.5 & PPMS \\
\hline 19 & $M$ & 42 & 4 & RRMS \\
\hline 20 & $\mathrm{~F}$ & 39 & 3 & RRMS \\
\hline 21 & $M$ & 22 & 5.5 & RRMS \\
\hline 22 & $\mathrm{~F}$ & 23 & 6.5 & RRMS \\
\hline
\end{tabular}

The patients tolerated the procedures well in general with manageable pain at the site of bone marrow aspiration in most patients. Headaches due to sterile meningitis and fever occurred in 12 of the 22 patients (54\%) but were easily treated with steroids and pain medications. One patient had nausea and vomiting that lasted for few hours and was easily controlled. Two patients were non evaluable for response due to loss of follow up. Fig. 1 shows an interesting phenomenon we observed on the MRIs demonstrating a significant decrease in the number and intensity of some of the demyelinated areas after the treatment.

16 of the 20 evaluable patients improved as assessed by their EDSS score (80\%). The responses were in general durable (Table 2). The improvement was 1 point on average on the EDSS score (Fig. 2). In many, it meant being able to ambulate or eat independently when they needed much more help in the past and that resulted in significant subjective quality of life improvement.

There were no other long term effects noted to prevent repeating the procedure where it was found to be useful. There were also no issues to prevent the use of other therapies especially the newer agents where the neurologist felt the necessity.

Table 2. Results

\begin{tabular}{|c|c|c|c|}
\hline $\begin{array}{l}\text { Patient } \\
\text { number }\end{array}$ & $\begin{array}{c}\text { Pre } \\
\text { treatment } \\
\text { EDSS }\end{array}$ & $\begin{array}{c}\text { Post } \\
\text { treatment } \\
\text { EDSS }\end{array}$ & $\begin{array}{l}\text { Duration of } \\
\text { response } \\
\text { (in years) }\end{array}$ \\
\hline 1 & 6 & 4.5 & 2 \\
\hline \multicolumn{4}{|l|}{2} \\
\hline 3 & 2.5 & 1 & 2 \\
\hline 4 & 3 & 1.5 & 3 \\
\hline 5 & 1.5 & 0 & 4 \\
\hline 6 & 6 & 5.5 & \\
\hline 7 & 7 & 6 & 1.5 \\
\hline 8 & 7.5 & 5.5 & 1 \\
\hline 9 & 7.5 & 7.5 & 2 \\
\hline 10 & 8.5 & 8 & 1 \\
\hline 11 & 8.5 & 8.5 & \\
\hline 12 & 8.5 & 9 & 5 \\
\hline 13 & 6.5 & 4 & 1 \\
\hline 14 & 7.5 & 7.5 & 1 \\
\hline 15 & 2.5 & 1 & 1 \\
\hline 16 & 1 & 0 & 1 \\
\hline 17 & 2.5 & 0 & 1 \\
\hline 18 & 4.5 & 2 & 0.5 \\
\hline 19 & 4 & & \\
\hline 20 & 3 & 2 & 1 \\
\hline 21 & 5.5 & 5 & 0.5 \\
\hline 22 & 6.5 & 5 & 0.8 \\
\hline
\end{tabular}



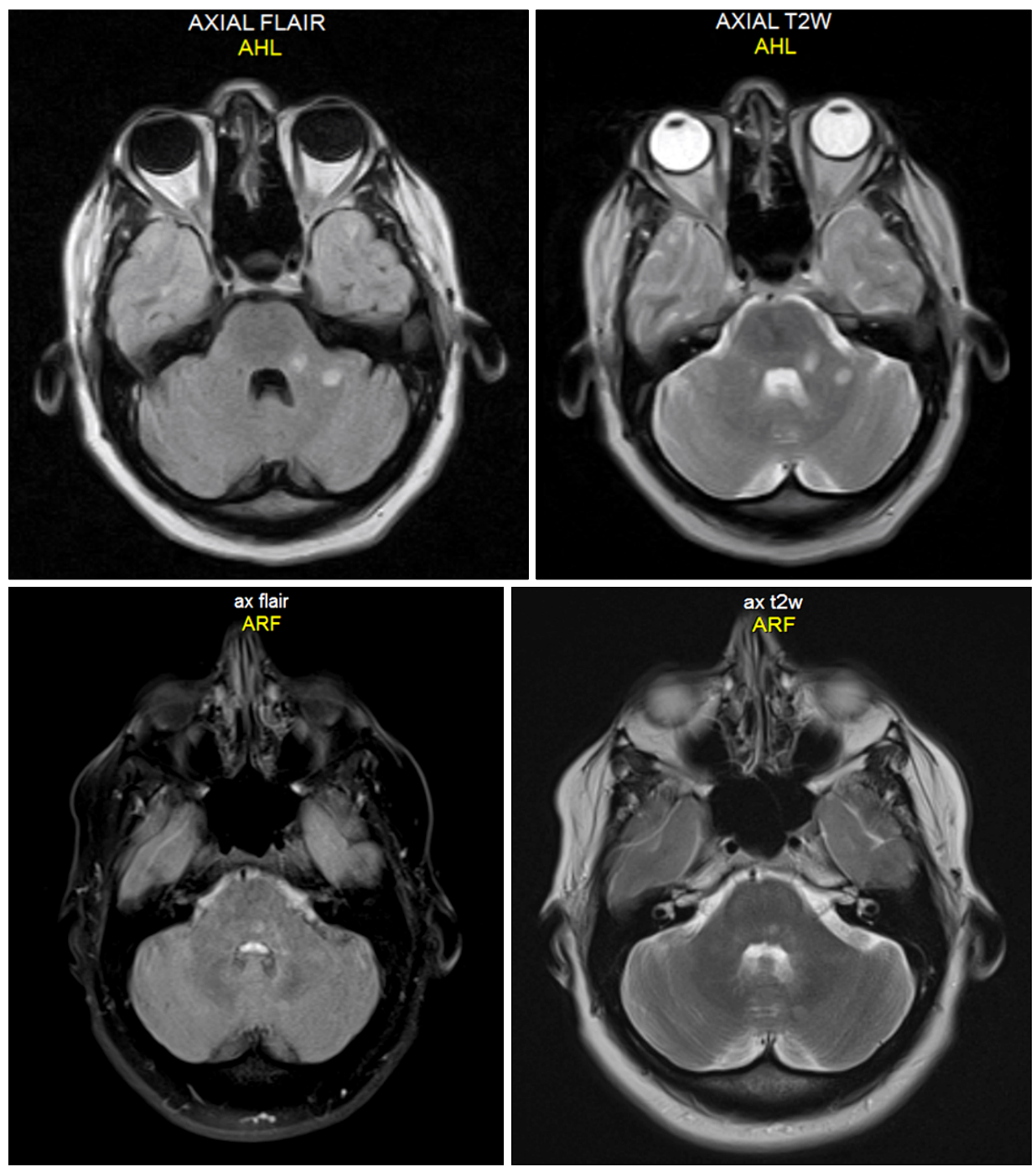

Fig. 1. MRI of the brain showing the demyelination lesions before (top row) and after treatment (bottom row). The photos show the areas of the demyelination process to have improved over time.

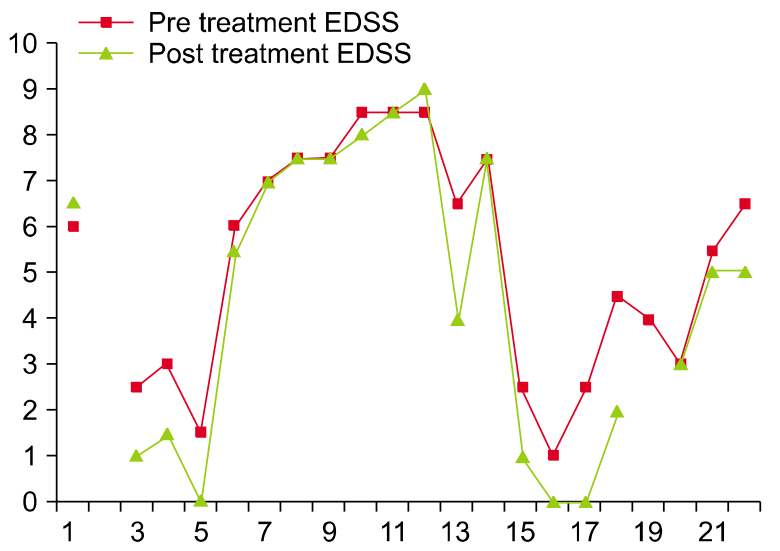

Fig. 2. The pre and post EDSS scores as determined before and after treatment.
The response seems to vary from one patient to another as reported in other studies $(16,19)$ although it was our impression that the lesser the symptoms and their duration, the better the response in general (Table 2). The pathobiology of the disease makes it a logical conclusion given the fact that the inflammatory process is at least partially reversible up to the point of neuronal death where it becomes permanent.

\section{Statistical Analysis}

When comparing the pre and post EDSS scores, we found a trend towards improvement of the patients' functional capacity but the $\mathrm{p}$ value did not reach statistical significance probably due to our sample size and the study design as a phase II rather than a randomized trial. However, comparing the expected EDSS scores to the post treatment values, the $\mathrm{p}$ value and statistical significance 
improved significantly to a two-tailed $\mathrm{p}$ value of 0.04 . One has to keep in mind that the patients' progression can be unpredictable and does not usually follow a clear pattern.

The $95 \%$ confidence interval was -1.003 to 2.653 with a mean of 5.3 before treatment and 4.475 after therapy. The standard deviation widened from 2.5 to 3.2 post treatment indicating the difference in responses seen among patients. Even when the expected worsening is accounted for, the improvement shows a wide standard deviation again reflecting the variations among the patients' responses.

\section{Discussion}

The available treatments for MS usually aim at providing symptom relief with no or little effect on the intricate pathobiology of the disease, effector cells and intermediary molecules. There is no available cure but rather calming treatments to maintain function for as long as possible.

Due to the presence of a big gap in the treatment of MS and the major permanent disability that results, we felt the need to look for alternative ways and new approaches to treat this disease.To assess the patients, we used a validated assessment tool which is the EDSS score.

Several growth factors like G-CSF, Stem Cell Factor (SCF), the fibroblast growth factor (FGF) and others may help reduce the ischemia and enhance the migration and proliferation of the stem cells. There is evidence that the growth factors like the ones released from the stem cells may help improve neuronal regeneration (24).

The transplanted bone marrow cells infiltrate the brain and may help regenerate new elements or combat the neurodegenerative process, fibrosis and oxidative insults. Several studies reported significant improvement among patients with neurodegenerative conditions with no significant adverse events. In this study, we report a significant improvement in $80 \%$ of the patients treated for progressive MS with minor adverse events and great tolerability as reported by other groups $(16,19,25)$. Chaitinya et al. and others used BM-MNSCs to treat Cerebral Palsey, giving five intrathecal injections, they reported significant clinical improvement with no safety issues (16, 26).

The Bone marrow contains several types of progenitors including the hematopoietic and mesenchymal stem cells in addition to mature differentiated cells. There are several likely mechanisms of action. The BM-MNSCs seem to concentrate in injured areas and act through paracrine effects to reduce the inflammatory process. The BM-MNSCs also contribute to vascular repair and stimulate en- dogenous stem cells to proliferate and contribute to the repair process. The BM-MNSCs may generate neurons; counteract fibrosis and oxidation (27-29).

\section{Conclusion}

There are several auto-immune conditions that reportedly showed a good response to stem cell therapy using different methods. These conditions include Crohn's disease, graft versus host disease (GvHD), lupus, and others (30).

In this report, we summarized the course of 22 patients treated with bone marrow derived stem cells. The cells were given using intravenous and intrathecal routes. Our list of patients adds to the current evidence that the BM-MNSCs are safe and partially effective in many patients with MS.

Those cells can be combined with other immunotherapeutic approaches as well as growth factors in the future and tested extensively in phase III studies to demonstrate and prove their efficacy beyond doubt. The injections could be repeated theoretically to sustain the improvement or add to it over time should the patient benefit from this approach. It is not clear at this time what is the best number of injections, the injection schedule, or whether it is beneficial to add growth factors to maximize this effect.

It is also important for the regulatory agencies to keep up with the progress being made in the stem cell science and assist in its proper implementation and benefit. A very large number of hurdles remain but we have to keep in mind the special aspects of this line of therapy requiring new methodologies to keep abiding by the scientific methods and medical ethics. The very concept of cellular therapy entails a fundamental new look at our concepts of pharmacodynamics and pharmacokinetics along with new roles for using new differentiation growth factors and special in vivo microenvironments reaching a re-definition of the different phases of the clinical studies.

Cell therapy may be the future pathway to solve a large number of debilitating and disabling situations if we can channel this effort into the correct paths.

\section{Acknowledgement}

We acknowledge the contribution of our patients and their families to this work.

\section{Potential conflict of interest}

The authors have no conflicting financial interest. 


\section{References}

1. Compston A, Coles A. Multiple sclerosis. Lancet 2008; 372:1502-1517

2. Weinshenker BG. Epidemiology of multiple sclerosis. Neurol Clin 1996;142:291-308

3. Barkhof F, Bruck W, De Groot CJ, Bergers E, Hulshof S, Geurts J, Polman CH, van der Valk P. Remyelinated lesions in multiple sclerosis: magnetic resonance image appearance. Arch Neurol 2003;60:1073-1081

4. Navikas V, Link H. Review: cytokines and the pathogenesis of multiple sclerosis. J Neurosci Res 1996;45:322-333

5. Kingwell K. Disease mechanisms in MS: informing tactics to combat MS. Nature Reviews Neurology 2012;8:589

6. Chang A, Smith MC, Yin X, Fox RJ, Staugaitis SM, Trapp BD. Neurogenesis in the chronic lesions of multiple sclerosis. Brain 2008;131:2366-2375

7. Calabresi PA. Diagnosis and management of multiple sclerosis. Am Fam Physician 2004;70:1935-1944

8. Lublin FD, Reingold SC. Defining the clinical course of multiple sclerosis: results of an international survey. National multiple sclerosis society (USA) advisory committee on clinical trials of new agents in multiple sclerosis. Neurology 1996;46:907-911

9. Franklin RJ, Ffrench-Constant C. Remyelination in the CNS: from biology to therapy. Nat Rev Neurosci 2008; 9:839-855

10. Castro-Borrero W, Graves D, Frohman TC, Flores AB, Hardeman P, Logan D, Orchard M, Greenberg B, Frohman EM. Current and emerging therapies in multiple sclerosis: a systematic review. Ther Adv Neurol Disord 2012;5: 205-220

11. CAMMS223 Trial Investigators, Coles AJ, Compston DA, Selmaj KW, Lake SL, Moran S, Margolin DH, Norris K, Tandon PK. Alemtuzumab vs. interferon beta-1a in early multiple sclerosis. N Engl J Med 2008;359:1786-1801

12. Bielekova B. Daclizumab therapy for multiple sclerosis. Neurotherapeutics 2013;10:55-67

13. Corboy JR, Miravalle AA. Emerging therapies for treatment of multiple sclerosis. J Inflamm Res 2010;3:53-59

14. Lunn JS, Sakowski SA, Federici T, Glass JD, Boulis NM, Feldman EL. Stem cell technology for the study and treatment of motor neuron diseases. Regen Med 2011;6:201-213

15. Dantuma E, Merchant S, Sugaya K. Stem cells for the treatment of neurodegenerative diseases. Stem Cell Res Ther 2010;1:37

16. Abi Chahine NH, Wehbe TW, Hilal RA, Zoghbi VV, Melki AE, Habib EB. Treatment of cerebral palsy with stem cells: a report of 17 cases. Int J Stem Cells 2016;9:90-95

17. Trounson A. New perspectives in human stem cell therapeutic research. BMC Med 2009;7:29

18. Abi Chahine NH, Abou Saad ES, Melki SGE. How to maximize the success of stem cell autografts for neuroregeneration. J Stem Cell Res Ther 2015;5:271

19. Sharma A, Gokulchandran N, Chopra G, Kulkarni P, Lohia M, Badhe P, Jacob VC. Administration of autologous bone marrow-derived mononuclear cells in children with incurable neurological disorders and injury is safe and improves their quality of life. Cell Transplant 2012;21 Suppl $1:$ S79-S90

20. Kuban JL, Hecht AB, Onderdonk T, O'Shea MN. Understanding the underlying beneficial biology of stem cells and the development and validation of more relevant animal models is required. Paneth Pediatr Res 2010;67: 95-101

21. Payne N, Siatskas C, Barnard A, Bernard CC. The prospect of stem cells as multi-faceted purveyors of immune modulation, repair and regeneration in multiple sclerosis. Curr Stem Cell Res Ther 2011;6:50-62

22. Mezey E, Key S, Vogelsang G, Szalayova I, Lange GD, Crain B. Transplanted bone marrow generates new neurons in human brains. Proc Natl Acad Sci 2003;100:1364-1369

23. Connick P, Kolappan M, Crawley C, Webber DJ, Patani R, Michell AW, Du MQ, Luan SL, Altmann DR, Thompson AJ, Compston A, Scott MA, Miller DH, Chandran S. Autologous mesenchymal stem cells for the treatment of secondary progressive multiple sclerosis: an open-label phase 2a proof-of-concept study. Lancet Neurol 2012;11:150-156

24. Schäbitz WR, Krüger C, Pitzer C, Weber D, Laage R, Gassler N, Aronowski J, Mier W, Kirsch F, Dittgen T, Bach A, Sommer C, Schneider A. A neuroprotective function for the hematopoietic protein granulocyte-macrophage colony stimulating factor (GMCSF). J Cereb Blood Flow Metab 2008;28:29-43

25. Krause DS, Theise ND, Collector MI, Henegariu O, Hwang S, Gardner R, Neutzel S, Sharkis SJ. Multi-organ, multi-lineage engraftment by a single bone marrow-derived stem cell. Cell 2001;105:369-377

26. Purandare C, Shitole DG, Belle V, Kedari A, Bora N, Joshi M. Therapeutic potential of autologous stem cell transplantation for cerebral palsy. Case Rep Transplant 2012; 825289

27. Tse HF, Kwong YL, Chan JK, Lo G, Ho CL, Lau CP. Angiogenesis in ischaemic myocardium by intramyocardial autologous bone marrow mononuclear cell implantation. Lancet 2003;361:47-49

28. Nakano-Doi A, Nakagomi T, Fujikawa M, Nakagomi N, Kubo S, Lu S, Yoshikawa H, Soma T, Taguchi A, Matsuyama T. Bone marrow mononuclear cells promote proliferation of endogenous neural stem cells through vascular niches after cerebral infarction. Stem Cells 2010; 28:1292-1302

29. Amin AH, Abd Elmageed ZY, Nair D, Partyka MI, Kadowitz PJ, Belmadani S, Matrougui K. Modified multipotent stromal cells with epidermal growth factor restore vasculogenesis and blood flow in ischemic hind-limb of type II diabetic mice. Lab Invest 2010;90:985-996

30. Mimeault M, Hauke R, Batra SK. Stem cells: a revolution in therapeutics-recent advances in stem cell biology and their therapeutic applications in regenerative medicine and cancer therapies. Clin Pharmacol Ther 2007;82:252-264 